\title{
A Range-Only Tracking Algorithm for Wireless Sensor Networks
}

\author{
Evangelos B. Mazomenos Jeffrey S. Reeve Neil M. White \\ School of Electronics \& Computer Science, University of Southampton \\ Southampton, S017 1BJ, UK \\ Phone:+44 2380495996 \\ \{ebm07r, jsr, nmw\}@ecs.soton.ac.uk
}

\begin{abstract}
Over the last few years, target tracking in wireless sensor networks has become a topic of particular interest. This paper presents a tracking system intended for deployment in distributed Wireless Sensor Networks. The approach is inspired from the concept of deploying sensor nodes in an adhoc manner and based on the aggregate amount of information they provide, perform tracking of mobile objects. Static sensor nodes, with known locations, act as anchor nodes providing range-only measurements at each time step. The proposed system is modeled using a nonlinear state-space model which reflects a real world tracking scenario. The batch of range measurements made available at each sampling step, is used to estimate the target's desired kinematic properties. In order to infer the state of the target at each time step, a Particle Filter algorithm has been designed to approximate the required posterior distribution of the state vector. The system's operation was simulated and execution examples demonstrate the algorithm's accuracy as well as the ability to effectively cope with manoeuvring targets.
\end{abstract}

\section{Introduction}

The ability to accurately estimate the trajectory of a ground moving target is of great importance for a number of application areas. Military surveillance systems, industrial, personnel and wildlife monitoring systems require tracking schemes, capable of inferring the kinematic characteristics (eg. position, velocity, acceleration) of one or more targets of interest. To infer the target's kinematic characteristics, a certain modality must be measured and mathematically associated to them. An example is the Received Signal Strength Indication (RSSI) measured in a sensor, which can be associated with the relative distance between the sensor and the signal source. Another such modality is the Doppler effect which is extensively used in RADAR systems.

Wireless Sensor Networks (WSNs), constitute of a num- ber of low power hardware platforms known as "motes" [11], capable of performing limited computation, sensing the environment and communicating through a wireless link. WSNs are considered to be suitable for a broad spectrum of application areas. Typical application examples for WSNs, include environmental and industrial monitoring, motion detection and target tracking, smart home environments and health applications [1].

In recent years, tracking ground mobile targets with WSN has attracted considerable research interest [10, 17]. In [10] a military surveillance and tracking system, formed in a large scale ( $>200$ nodes) WSN, is presented. The system's mission is to detect a military target which intrudes into the monitored area and effectively classify the intruder, based on readings received by three sensor types. A similar scenario is considered by Arora et.al in [2], where a pulse radar among other sensors is incorporated on WSN nodes to provide classifying measurements.

This paper discusses a novel approach to implementing target tracking in a WSN. It differs from from other approaches where the tracking algorithm runs on single node data $[12,17]$. Our algorithm relies on accumulated data from a small number of nodes. The results obtained reveal that, very good accuracy can be achieved by employing a low complexity Particle Filter tracking algorithm and combining data from a number of nodes.

The remainder of the paper is structured as follows. Section 2 presents the related work in the area, followed by the problem formulation in Section 3. Section 4 describes the range-only tracking algorithm that is proposed in this paper and simulations evaluate its performance in the sequel. Concluding remarks are discussed in the final section.

\section{Background}

Particle Filters $(\mathrm{PF})$ are a class of recursive Bayesian estimation filters, based on Sequential Monte Carlo methods [9]. PF have proved, to be able to handle efficiently nonlinear system models which are often utilized in tracking sce- 
narios. In several tracking applications PF have performed substantially better than other known nonlinear filters like the Extended Kalman Filter (EKF) or Grid Based Methods [3]. PF approximation of the posterior probability density function (pdf) is achieved by representing the respective pdf with a set of particles and their corresponding weights. Particles are sampled from a proposal density function according to the Sequential Importance Sampling (SIS) method. $\mathrm{PF}$ have been investigated for implementation is a vast number of application areas. From tracking and navigation to econometric estimation [13]. The flexibility they offer renders PF an ideal alternative in estimation problems involving nonlinear models.

As stated earlier, tracking ground mobile targets using a network of cooperating sensor nodes has attracted substantial research interest. Subsequently, PF have been proposed in scenarios where tracking is performed by low power, resource constrained WSN nodes. One of the most important aspects of implementing PF on WSNs hardware is their computational complexity which sometimes can be prohibitive, for implementation in hardware with limited processing ability. The complexity of the PF algorithms increases if complex state space equations are chosen to represent the system model, or a large number of particles is chosen to represent the pdf.

Examples of PF algorithms for tracking in WSNs are described at $[15,14,7]$. Two distributed PF tracking algorithms for sensor networks are presented in $[5,6]$. Each node maintains a current belief of the posterior distribution of the state and updates this belief based on data from its sensors. This candidate state is then forwarded to the next tracking node. This approach results in a high communication overhead and requires the execution of complex computations by resource limited nodes. Tracking with a network of range and radar sensors is proposed in [4].

Range-Only Tracking is considered to be a hard problem, mostly because global system observability may not exist [16]. Nevertheless, tracking of mobile targets with range-only measurements can be achieved if more than one observers provide range estimates. This particular concept reflects the fundamental concept of WSNs. Multiple nodes act as observers, providing a range signature of the target. A single node would be incapable of performing any kind of tracking with range measurements. On the contrary, a group of nodes can provide an adequate amount of information and ensure observability, for an effective range-only tracking algorithm to be designed. Recently in [12] a distributed hybrid tracking algorithm is proposed, which combines a PF and a Probabilistic Data Association Filter. The complexity of this algorithm is relatively high. The authors belief is that, similar accuracy can be achieved using lower complexity algorithms.

\section{Problem Formulation}

We form our system using a state-space model. An arbitrarily deployed network consisting of $N_{s}$ sensor nodes is considered. The state vector contains the object's planar coordinates $(\mathbf{x}, \mathbf{y})$ and velocities $\left(\mathbf{v}_{x}, \mathbf{v}_{y}\right)$ along the $\mathbf{x}$-axis and the y-axis. Thus:

$$
\mathbf{x}=\left[\begin{array}{llll}
x & v_{x} & y & v_{y}
\end{array}\right]^{T} .
$$

The state of the target evolves in time according to the following discrete time stochastic model:

$$
\mathbf{x}_{k}=\mathbf{F} \mathbf{x}_{k-1}+\boldsymbol{\Gamma} \mathbf{w}_{k-1}
$$

The measurements, made available at each time step, are associated to the state vector via the measurements equation:

$$
\mathbf{z}_{k}=\mathbf{h}\left(\mathbf{x}_{k}, \mathbf{v}_{k}\right)
$$

- where time index $k$ is discrete: $k=1,2 \ldots, K$

- the matrices $\mathbf{F}$ and $\boldsymbol{\Gamma}$ are defined as follows:

$$
\mathbf{F}=\left[\begin{array}{cccc}
1 & T_{s} & 0 & 0 \\
0 & 1 & 0 & 0 \\
0 & 0 & 1 & T_{s} \\
0 & 0 & 0 & 1
\end{array}\right]
$$

and,

$$
\boldsymbol{\Gamma}=\left[\begin{array}{cc}
T_{s}^{2} / 2 & 0 \\
0 & T_{s}^{2} / 2 \\
T_{s} & 0 \\
0 & T_{s}
\end{array}\right]
$$

- $T_{s}$ : is the sampling period

- $\mathbf{w}_{k-1}$ : represents the system noise and, $\mathbf{v}_{k}$ : represents the measurements noise. Both noise sources are assumed to have known distributions

- In our case we consider that range measurements from a number of $N_{s}$ static sensors become available at each time step $k=1 \ldots K$. Hence, the measurements vector $\mathbf{z}_{n, k}$, where $n=1, \ldots, N_{s}$ is given from:

$$
\mathbf{z}_{n, k}=\left[\begin{array}{c}
\sqrt{\left(y_{k}-y_{1}\right)^{2}+\left(x_{k}-x_{1}\right)^{2}} \\
\sqrt{\left(y_{k}-y_{2}\right)^{2}+\left(x_{k}-x_{2}\right)^{2}} \\
\sqrt{\left(y_{k}-y_{3}\right)^{2}+\left(x_{k}-x_{3}\right)^{2}} \\
\vdots \\
\sqrt{\left(y_{k}-y_{N_{s}}\right)^{2}+\left(x_{k}-x_{N_{s}}\right)^{2}}
\end{array}\right]
$$


where $\left(x_{k}, y_{k}\right)$ is the $x$ and $y$ coordinate of the target at time $k$.

We denote as $\mathbf{Z}_{k}$ the vector of range estimates from all the available sensors up to time $k$.

In the above definition of the nonlinear system, only a single mobile target is considered for monitoring. The proposed system model can easily be extended to include multiple targets.

The posterior probability density function (pdf) of the state $p\left(\mathbf{x}_{k} \mid \mathbf{Z}_{k}\right)$ must be constructed in order to infer the system's state vector, thus the target's position and velocity.

\section{ROT-PF Algorithm}

In this section we provide insight into the Particle Filter algorithm we employ, in order to solve the problem analyzed in the previous section. Particle Filters produce an approximation of the required pdf using a set of particles $\left\{\mathbf{x}_{k}^{i}, i=1,2, \ldots, N\right\}$ and associated weights $\left\{w_{k}^{i}, i=\right.$ $1,2, \ldots, N\}$. Particles are initialized from an initial distribution which describes the uncertainty regarding the object's initial state. Henceforth, particles are sampled from a proposal distribution $q\left(\mathbf{x}_{k} \mid \mathbf{x}_{k-1}\right)$. The importance weights are given from the following relationship:

$$
w_{k}^{i} \propto \frac{p\left(\mathbf{X}_{k}^{i} \mid \mathbf{Z}_{k}\right)}{q\left(\mathbf{X}_{k}^{i} \mid \mathbf{Z}_{k}\right)}
$$

which yields the weights update equation,

$$
w_{k}^{i} \propto w_{k-1}^{i} \frac{p\left(\mathbf{z}_{k} \mid \mathbf{x}_{k}^{i}\right) p\left(\mathbf{x}_{k}^{i} \mid \mathbf{x}_{k-1}^{i}\right)}{q\left(\mathbf{x}_{k}^{i} \mid \mathbf{x}_{k-1}^{i}, \mathbf{z}_{k}\right)}
$$

Our choice of proposal distribution is the transitional prior $p\left(\mathbf{x}_{k} \mid \mathbf{x}_{k-1}\right)$. In this case, weights are calculated from the likelihood function.

$$
w_{k}^{i}=p\left(\mathbf{z}_{n, k} \mid \mathbf{x}_{k}^{i}\right)
$$

hence the weights update equation is given by:

$$
w_{k}^{i} \propto w_{k-1}^{i} p\left(\mathbf{z}_{n, k} \mid \mathbf{x}_{k}^{i}\right)
$$

With the reception of a new measurement vector at each sampling time the weights are updated and propagated to the next time step.

At time $k$, an approximation of the posterior density of the state is given by:

$$
p\left(\mathrm{X}_{k} \mid \mathrm{Z}_{k}\right) \approx \sum_{i=1}^{N} w_{k}^{i} \delta\left(\mathrm{X}_{k}-\mathrm{X}_{k}^{i}\right)
$$

Based on the above representation of the state pdf an estimate for the state at time $k$ according to the MMSE criterion is:

$$
\hat{\mathbf{x}_{k}}=\sum_{i=1}^{N} \mathbf{x}_{k}^{i} w_{k}^{i}
$$

One common problem that PF algorithms encounter is the degeneracy problem. In practical terms, this means that after a certain number of iterations, all but one particles will have negligible weights. The degeneracy problem causes a substantial amount of computation, to update particles, to be devoted to particles that have a negligible contribution to the approximation of the posterior pdf. Degeneracy can be identified by introducing the effective sample size $N_{\text {eff }}$ defined as follows:

$$
N_{e f f}=\frac{1}{\sum_{i=1}^{N}\left(w_{k}^{i}\right)^{2}}
$$

To avoid the degeneracy effect in our ROT-PF algorithm we compare $N_{\text {eff }}$ with a certain resampling threshold $N_{t h r}$ and if resampling is needed, a residual resampling step is carried out according to [8]. Resampling alleviates the degeneracy effect by eliminating particles with negligible weights, while particles with high importance weights are propagated. We choose to perform resampling at every time step.

An iteration of the ROT - PF algorithm at time $k$ is summarized in Table 1:

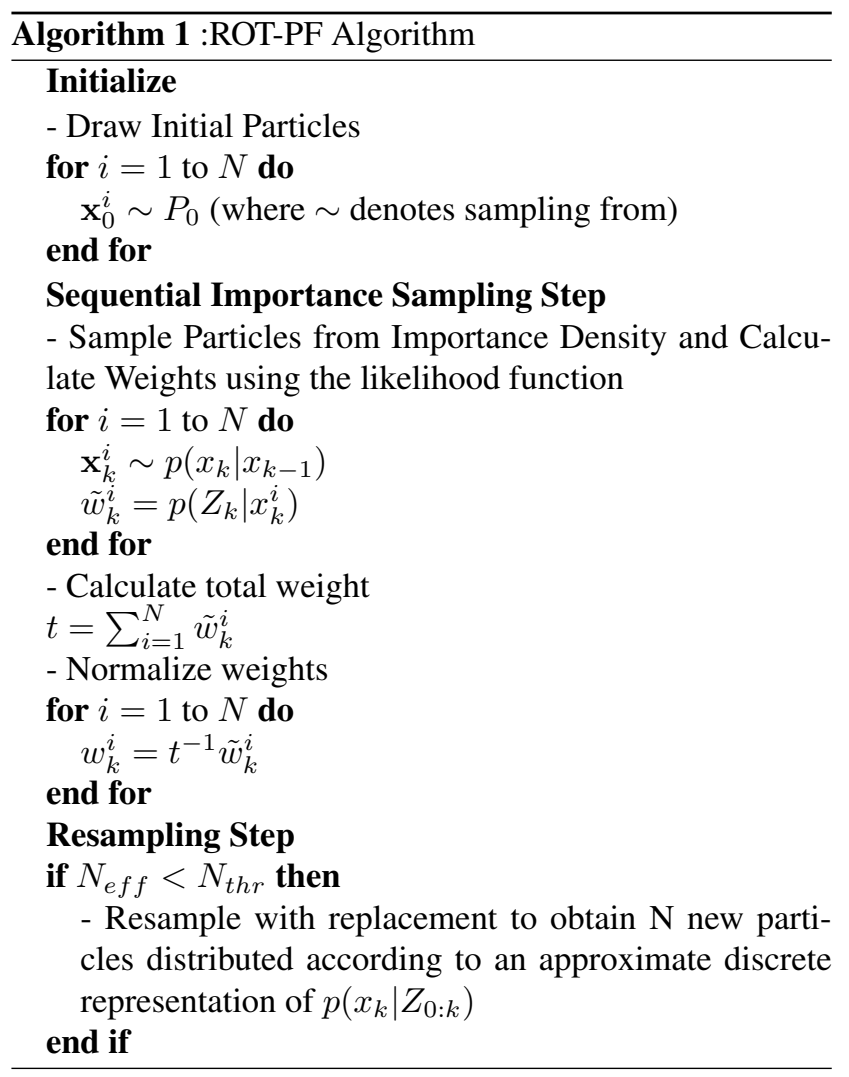

\section{Simulations}

Simulations have been conducted to illustrate the performance of our tracking algorithm. We evaluate the perfor- 
mance of our algorithm and predict the accuracy that the tracking system is capable of achieving. A single mobile target is the object of interest and four anchor nodes provide range measurements. Several trajectories were simulated in order to evaluate the algorithms performance in different motion patterns. Manoeuvring targets were also considered. The authors envisage a system where nodes are spread in an ad-hoc manner, localize themselves and then initiate tracking whenever this is requested. These examples simulate only the tracking operation and do not make any implications regarding the nodes deployment or how the system became aware of the anchor nodes coordinates.

\subsection{A scenario of high initial uncertainty and heavy clutter}

A WSN, deployed in the following way, was considered in the simulation examples. Four anchor nodes have been deployed to provide ;range-only measurements at each time step. The coordinates of the anchor nodes are; $s_{1}=$ $[20,20], s_{2}=[20,60], s_{3}=[80,20], s_{4}=[80,60]$. It has to be stated that the position of the anchor nodes should be known for the tracking process. However this does not implies that the nodes should be placed at predefined positions.

A single ground mobile targets is considered in our scenario. The target's state-vector evolves according to Equation 2 and the measurement are associated with the state through Equation 6. The sampling period is set to $T_{s}=1$ and the number of particles used in every iteration of the algorithm is $N=500$. The process and measurement additive noise sources are presumed to follow zero mean Gaussian distributions. The state noise covariance is $q=0.05 I_{2}$ and the measurement noise covariance is $r=0.5 I_{4}$ (where $I$ denotes the identity matrix). The target's initial state is $\mathbf{x}_{0}=\left[\begin{array}{llll}10 & 0.4 & 15 & 0.3\end{array}\right]$. Initial particles are sampled from a Gaussian distribution with zero mean and covariance matrix $P_{0}$ which was defined as;

$$
\mathbf{P}_{\mathbf{0}}=\left[\begin{array}{cccc}
2^{2} & 0 & 0 & 0 \\
0 & 0.5^{2} & 0 & 0 \\
0 & 0 & 3^{2} & 0 \\
0 & 0 & 0 & 0.5^{2}
\end{array}\right]
$$

The simulation time was $T=75$ steps. The system was simulated for a total of $L=100$ times. An exemplar run is illustrated in Fig. 1 - trajectory and Fig2 - velocity.

In order to quantify the accuracy of the ROT-PF algorithm, the root mean square error was calculated for every run;

$$
\mathbf{R M S E}=\sqrt{\frac{1}{T} \sum_{t=1}^{T}\left(x-x_{e s t}\right)^{2}+\left(y-y_{e s t}\right)^{2}}
$$

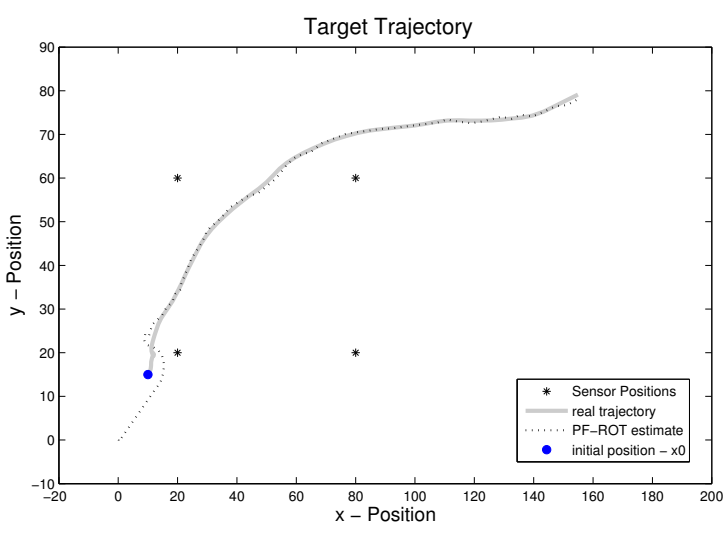

Figure 1. True and Filtered target trajectory

The simulated system presents high uncertainty regarding the target's initial state. Moreover both the state and measurement noise sources present high variance. We opted to choose a system, subjected to high clutter, in order to demonstrate the ability of the ROT-PF algorithm to effectively track the target's state, regardless of the initial uncertainty being high and the intense level of noise that corrupts the system's state and measurement model.

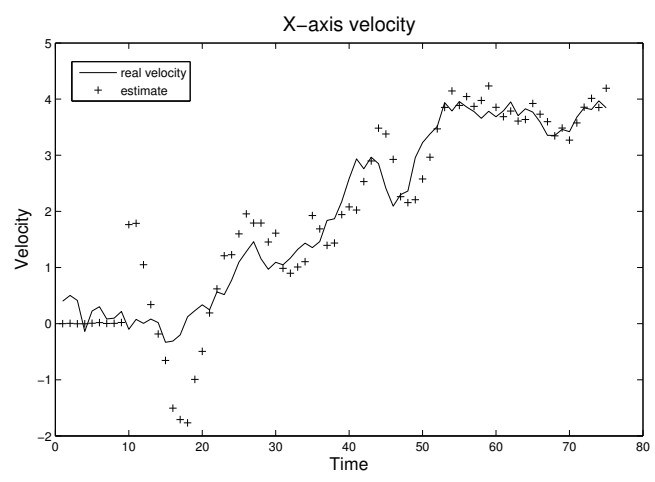

(a) $\mathrm{X}$-axis velocity estimation

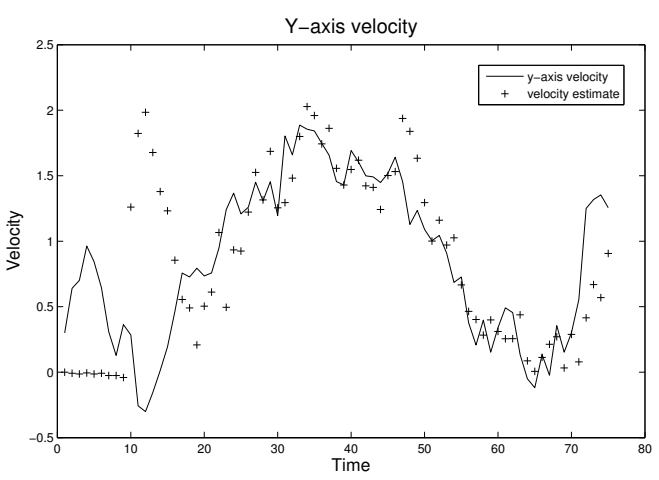

(b) y-axis velocity estimation

Figure 2. Two-axis velocity estimation 
Fig. 3(a) illustrates the RMSE for a total of 100 runs of the system described. In the vast majority of executions the RMSE remains under 10 at absolutely satisfactory limits. Only in a small fraction (5-6\%) of the executions, the RMSE increases up to unacceptable levels. The high level of initial uncertainty is responsible for the poor performance of the ROT-PF algorithm in these runs. Furthermore, when considering the algorithm's performance, the small number of particles $(\mathrm{N}=500)$, must be taken under consideration. By increasing the number of particles we can achieve better accuracy in terms of RMSE.

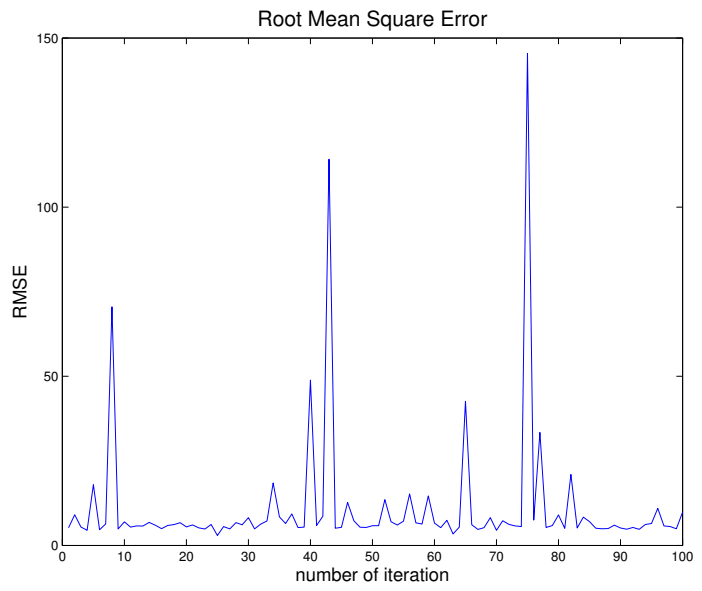

(a) RMSE for 100 simulation runs $-\mu_{0}=0$

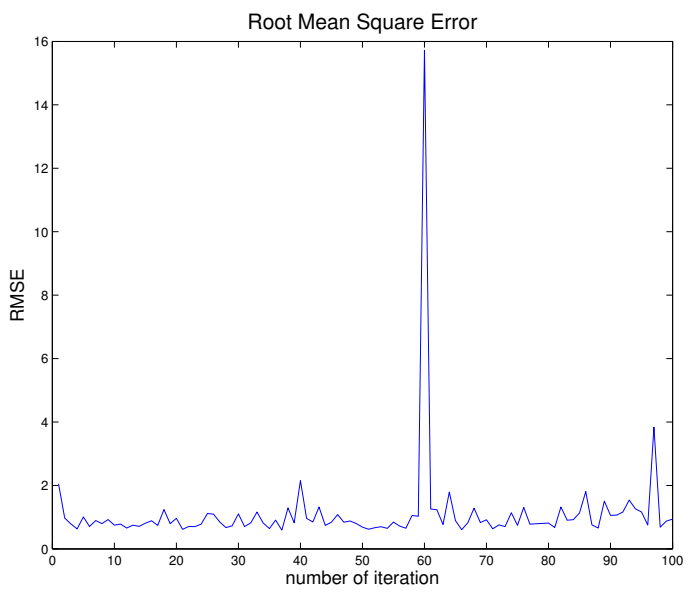

(b) RMSE for 100 simulation runs - $\mu_{0}=x_{0}+\mathcal{N}\left(0,1.5^{2}\right)$

Figure 3. RMSE for 100 simulation runs

In Fig. 3(b) the same system, as previously was simulated for 100 runs. For this particular set of executions the uncertainty regarding the initial state was reduced. The initial particles were sampled from a Gaussian distribution with $\mu_{0}=x_{0}+\mathcal{N}\left(0,1.5^{2}\right)$. From Fig $3(\mathrm{~b})$ it is clear that the RMSE is drastically improved and kept below 20 in all executions.

\subsection{Manoeuvring Targets}

In this section we illustrate the capability of the ROT-PF tracking algorithm to handle manoeuvring targets, as this is normally the case for real world scenarios. Fig.4(a) and Fig 4(b) present an example of how accurate the ROT-PF performs in the presence of a manoeuvring target. The results are quite promising.
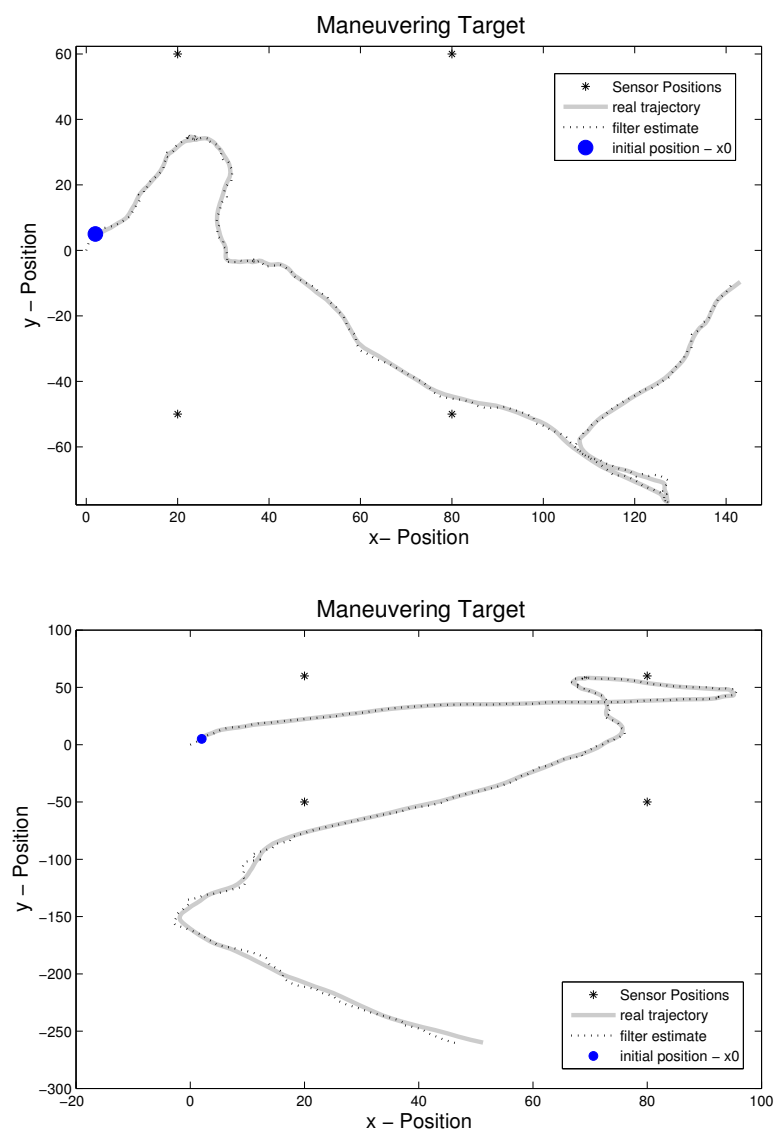

Figure 4. Manoeuvring Target Tracking

\section{Conclusions and Future Work}

In this paper, a novel target tracking algorithm intended for implementation in WSNs was presented. The algorithm is based on Particle Filters and is designed by taking into consideration a nonlinear system state-space model. The tracking operation is carried out by considering solely range measurements to be available from the WSN. The rangeonly nature of the systems renders it flexible for deployment in a broad spectrum of applications. A number of 
ranging techniques can provide range measurements with good accuracy, based on different modalities. Simulations produced prominent and solid results, revealing good performance in the majority of the scenarios considered. An important aspect of the system presented is that, good accuracy is possible while the number of particles that are sampled is relatively small. Thus, different to other approaches, the proposed system requires a small number of particles for estimating the required posterior pdf. The RMSE was calculated over a number of simulations to quantify the level of accuracy achieved. Similar accuracy, to approaches which employ more complicated algorithms, was achieved. Initial uncertainty regarding the targets state, at the beginning of the tracking operation, has proved to have an effect on the system's accuracy. Finally simulations justified the ability of the system to handle efficiently manoeuvring targets. Future research includes the investigation of more complex system models, particularly in order to best describe the target's motion. Including targets that manoeuvre in higher speed without increasing the complexity and the computational burden of the tracking algorithm, will be the authors future objective. Ultimately, this system is intended for deployment in actual WSN hardware in order to verify the simulation results in real world experiments.

\section{References}

[1] I. F. Akyildiz, W. Su, Y. Sankarasubramaniam, and E. Cayirci. Wireless sensor networks: a survey. Comput. Netw., 38(4):393-422, 2002.

[2] A. Arora, P. Dutta, S. Bapat, V. Kulathumani, H. Zhang, V. Naik, V. Mittal, H. Cao, M. Demirbas, M. Gouda, Y. Choi, T. Herman, S. Kulkarni, U. Arumugam, M. Nesterenko, A. Vora, and M. Miyashita. A line in the sand: a wireless sensor network for target detection, classification, and tracking. Comput. Netw., 46(5):605-634, 2004.

[3] M. Arulampalam, S. Maskell, N. Gordon, and T. Clapp. A tutorial on particle filters for online nonlinear/non-gaussian bayesian tracking. IEEE Transactions on Signal Processing, 50(2):174-188, Feb 2002.

[4] M. Borkar, V. Cevher, and J. McClellan. Estimating target state distributions in a distributed sensor network using a monte-carlo approach. In Machine Learning for Signal Processing, 2005 IEEE Workshop on, pages 305-310, Sept. 2005.

[5] M. Coates. Distributed particle filters for sensor networks. In Information Processing in Sensor Networks, 2004. IPSN 2004. Third International Symposium on, pages 99-107, April 2004.

[6] M. Coates and G. Ing. Sensor network particle filters: motes as particles. In Statistical Signal Processing, 2005 IEEE/SP 13th Workshop on, pages 1152-1157, July 2005.

[7] P. Djuric, M. Vemula, and M. Bugallo. Tracking with particle filtering in tertiary wireless sensor networks. In Acoustics, Speech, and Signal Processing, 2005. Proceedings.
(ICASSP '05). IEEE International Conference on, volume 4, pages 757-760, March 2005.

[8] A. Doucet, N. De Freitas, and N. Gordon. Sequential Monte Carlo Methods In Practice. Sringer, 2001.

[9] A. Doucet, N. Gordon, and V. Krishnamurthy. Particle filters for state estimation of jump markov linear systems. IEEE Transactions on Signal Processing, 49(3):613-624, Mar 2001.

[10] T. He, P. Vicaire, T. Yan, L. Luo, L. Gu, G. Zhou, R. Stoleru, Q. Cao, J. Stankovic, and T. Abdelzaher. Achieving realtime target tracking usingwireless sensor networks. In RealTime and Embedded Technology and Applications Symposium, 2006. Proceedings of the 12th IEEE, pages 37-48, 0407 April 2006.

[11] J. M. Kahn, R. H. Katz, and K. S. J. Pister. Next century challenges: mobile networking for "smart dust". In MobiCom '99: Proceedings of the 5th annual ACM/IEEE international conference on Mobile computing and networking, pages 271-278, New York, NY, USA, 1999. ACM.

[12] H. Ma and B.-H. Ng. Distributive target tracking in wireless sensor networks under measurement origin uncertainty. In Intelligent Sensors, Sensor Networks and Information, 2007. ISSNIP 2007. 3rd International Conference on, pages 299 304, Dec. 2007.

[13] B. Risfic, S. Arulampalam, and N. Gordon. Beyond the Kalman Filter - Particle Filters For Tracking Applications. Artech House Publishers, 2004.

[14] N. Shrivastava, R. M. U. Madhow, and S. Suri. Target tracking with binary proximity sensors: fundamental limits, minimal descriptions, and algorithms. In SenSys '06: Proceedings of the 4th international conference on Embedded networked sensor systems, pages 251-264, New York, NY, USA, 2006. ACM.

[15] J. Singh, U. Madhow, R. Kumar, S. Suri, and R. Cagley. Tracking multiple targets using binary proximity sensors. In IPSN '07: Proceedings of the 6th international conference on Information processing in sensor networks, pages 529538, New York, NY, USA, 2007. ACM.

[16] T. L. Song. Observability of target tracking with rangeonly measurements. Oceanic Engineering, IEEE Journal of, 24(3):383-387, Jul 1999.

[17] F. Zhao, J. Liu, J. Liu, L. Guibas, and J. Reich. Collaborative signal and information processing: an information-directed approach. Proceedings of the IEEE, 91(8):1199-1209, Aug. 2003. 